\title{
Generation of an Optimal Gait Trajectory for Biped Robots Using a Genetic Algorithm*
}

\author{
Jong Hyeon PARK ${ }^{* *}$ and Moosung $\mathrm{CHOI}^{* *}$
}

\begin{abstract}
This paper proposes a method that minimizes the energy consumption in the locomotion of a biped robot. A real-coded genetic algorithm is employed in order to search for the optimal locomotion pattern, and at the same time the optimal locations of the mass centers of the links that compose the biped robot. Since many of the essential characteristics of the human walking motion can be captured with a seven-link planar biped walking in the saggital plane, a 6-DOF biped robot that consists of seven links is used as the model used in the work. For trajectories of the robot in a single stride, fourth-order polynomials are used as their basis functions to approximate the locomotion gait. The coefficients of the polynomials are defined as design variables. For the optimal locations of the mass centers of the links, three variables are added to the design variables under the assumption that the left and right legs are identical. Simulations were performed to compare locomotion trajectories obtained with the genetic algorithm and the one obtained with the gravity-compensated inverted pendulum mode (GCIPM). They show that the proposed trajectory with the optimized mass centers significantly reduces the energy consumption, indicating that the proposed optimized method is a valuable tool in the design of biped robots.
\end{abstract}

Key Words: Biped Robot, Gait Trajectory, Saggital Plane, Genetic Algorithm, Optimization, Zero-Moment Point

\section{Introduction}

In general, mobile robots need to carry their own energy source in order to cover a large working area. Due to the limit in the amount of energy on board, a lower rate of energy consumption is highly desirable. Sometimes, large and powerful actuators are needed to track a certain trajectory, which requires more powerful and heavier batteries. A heavier robot in turn requires more powerful actuator to carry its own weight. This generates a vicious spiral for more power and energy. Thus, it is critical to minimize the energy consumption level in designing a robot and in generating the locomotion pattern.

Energy-efficient locomotion pattern results in a more natural walking motion ${ }^{(1)}$. Actually, a human naturally walks with a little energy consumption ${ }^{(2)}$. The locomotion trajectory is very critical in reducing the energy consumption of the robot and in making the walking more natural.

* Received 16th July, 2003 (No. 03-5092)

** Mechatronics Laboratory, School of Mechanical Engineering, Hanyang University, 17 Haengdang-Dong, Sungdong-Ku, Seoul 133-791, Korea.

E-mail: jongpark@hanyang.ac.kr
Many researchers have proposed methods to generate a gait trajectory for biped robots. Kajita and Tani ${ }^{(3)}$ proposed the linear inverted pendulum mode in order to generate reference trajectories. Park and Kim ${ }^{(4)}$ proposed the gravity-compensated inverted pendulum mode (GCIPM) and improved the locomotion stability by modelling the feet separately from the trunk. In these studies, much of the focus is on generating simple but stable trajectories for biped robots, but not on minimizing the energy consumption level. Various optimization schemes were proposed to generate energy-efficient trajectories for biped robots. Lee and Chen used the cubic B-splines for basis functions and the gradient-based algorithm to generate optimal trajectories ${ }^{(5)}$. Roussel et al. used the piecewise constant method to approximate the walking gait ${ }^{(6),(7)}$. Westervelt and Grizzle used an optimization package called DIRCOL, which implements an SQP algorithm and a variable number of cubic splines to approximate the state ${ }^{(8)}$. Capi et al. parameterized a robot state as a polynomial function and used a genetic algorithm for the optimiza$\operatorname{tion}^{(9)}$. Silva et al. searched for the optimal step length, hip height, link lengths and link masses, and so on, to minimize the energy consumption ${ }^{(10)}$. Chevallereau et al. 
searched for the optimal stride and period to generate optimal trajectories ${ }^{(11)}$.

All the methods mentioned above are focused on generating energy-efficient trajectories only after a biped robot is designed. However, designing of a robot and generating energy-efficient trajectories are closely linked together. Thus, they should be considered simultaneously, not sequentially. This paper proposes a method that minimizes the energy consumption by searching simultaneously for the optimal locations of the mass centers of the links and the energy-efficient trajectory. To the best of the author's knowledge, no work has ever been done in searching for optimal design parameters such as the position of the mass centers of the links, and at the same time the optimal gait trajectory. The locations of the mass centers are important in generating energy optimal trajectories. In a typical design phase of a robot, the positions of the motors, which are relatively heavier than other parts of the robot, are determined only by static design constraints. Once the optimal locations of the mass centers are found, the motors can be located at the desired location by the use of power transmission devices such as belts and pulleys.

In this paper, the optimal locations of the mass centers of the links are searched for during a stride of dynamic locomotion of the robot. Genetic algorithms are known to be efficient and robust in searching for a global solution in optimization $^{(9),(12)-(14)}$. The real-coded genetic algorithm is used due to its simplicity, speed, and easiness to deal with complex constraints.

This paper is organized as follows. In section 2, a 6DOF planar biped model is introduced. The constraints and numerical formulas for the optimization are described with brief explanations on the genetic algorithm in section 3. Simulations and the comparisons of three different gait trajectories generated by different methods including the GCIPM are covered in section 4. Finally, a few conclusions are drawn in section 5 .

\section{Biped-Robot Model}

\subsection{Biped-robot model in the saggital plane}

Since many of the essential characteristics of biped locomotion can be captured with a seven-link planar biped robot in the saggital plane ${ }^{(15)}$, a 6-DOF biped robot model that consists of seven links, as shown in Fig. 1, is used in this work. The physical parameters of the robot model are listed in Table 1. It is assumed that the mass of a link is concentrated at a single point on the link. It is also assumed that a complete locomotion cycle is divided in two phases: a single support phase in which one leg is in contact with the ground and the other leg swings forward, and an exchange of supports in which the two legs simultaneously trade their roles.

\subsection{Kinematic equations}

The kinematic relations for this biped-robot modelare

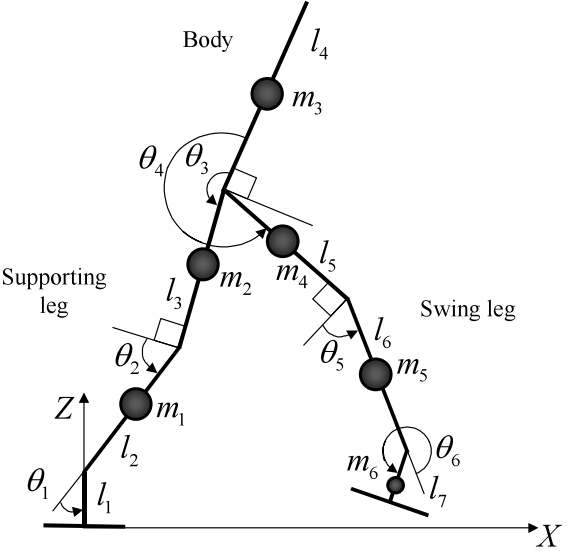

Fig. 1 A 6-DOF biped robot in the saggital plane

Table 1 Physical parameters of the biped robot model used

\begin{tabular}{c|cccc}
\hline link & 1 and 7 & 2 and 6 & 3 and 5 & 4 \\
\hline Length $(m)$ & 0.15 & 0.35 & 0.4 & 0.5 \\
Mass $(\mathrm{kg})$ & 1.0 & 5.0 & 2.5 & 6.0 \\
\hline
\end{tabular}

as follows.

$$
\begin{aligned}
x_{3}= & l_{2} s \theta_{1} \\
z_{3}= & l_{1}+l_{2} c \theta_{2} \\
x_{4}= & l_{2} s \theta_{1}-l_{3} c\left(\theta_{1}+\theta_{2}\right) \\
z_{4}= & l_{1}+l_{2} c \theta_{1}+l_{3} s\left(\theta_{1}+\theta_{2}\right) \\
x_{5}= & l_{2} s \theta_{1}-l_{3} c\left(\theta_{1}+\theta_{2}\right)+l_{5} c\left(\theta_{1}+\theta_{2}+\theta_{3}-\theta_{4}\right) \\
z_{5}= & l_{1}+l_{2} c \theta_{1}+l_{3} s\left(\theta_{1}+\theta_{2}\right)-l_{5} s\left(\theta_{1}+\theta_{2}+\theta_{3}-\theta_{4}\right) \\
x_{6}= & l_{2} s \theta_{1}-l_{3} c\left(\theta_{1}+\theta_{2}\right)+l_{5} c\left(\theta_{1}+\theta_{2}+\theta_{3}-\theta_{4}\right) \\
& -l_{6} s\left(\theta_{1}+\theta_{2}+\theta_{3}-\theta_{4}-\theta_{5}\right) \\
z_{6}= & l_{1}+l_{2} c \theta_{1}+l_{3} s\left(\theta_{1}+\theta_{2}\right)-l_{5} s\left(\theta_{1}+\theta_{2}+\theta_{3}-\theta_{4}\right) \\
& +l_{6} c\left(\theta_{1}+\theta_{2}+\theta_{3}-\theta_{4}-\theta_{5}\right) \\
x_{7}= & l_{2} s \theta_{1}-l_{3} c\left(\theta_{1}+\theta_{2}\right)+l_{5} c\left(\theta_{1}+\theta_{2}+\theta_{3}-\theta_{4}\right) \\
& -l_{6} s\left(\theta_{1}+\theta_{2}+\theta_{3}-\theta_{4}-\theta_{5}\right) \\
& -l_{7} s\left(\theta_{1}+\theta_{2}+\theta_{3}-\theta_{4}-\theta_{5}-\theta_{6}\right) \\
z_{7}= & l_{1}+l_{2} c \theta_{1}+l_{3} s\left(\theta_{1}+\theta_{2}\right)-l_{5} s\left(\theta_{1}+\theta_{2}+\theta_{3}-\theta_{4}\right) \\
& +l_{6} c\left(\theta_{1}+\theta_{2}+\theta_{3}-\theta_{4}-\theta_{5}\right) \\
& -l_{7} c\left(\theta_{1}+\theta_{2}+\theta_{3}-\theta_{4}-\theta_{5}-\theta_{6}\right) \\
x_{t}= & l_{2} s \theta_{1}-l_{3} c\left(\theta_{1}+\theta_{2}\right)+l_{4} s\left(\theta_{1}+\theta_{2}+\theta_{3}\right) \\
z_{t}= & l_{1}+l_{2} c \theta_{1}+l_{3} s\left(\theta_{1}+\theta_{2}\right)+l_{4} c\left(\theta_{1}+\theta_{2}+\theta_{3}\right)
\end{aligned}
$$

where $\left(x_{3}, z_{3}\right)$ and $\left(x_{5}, z_{5}\right)$ denote the positions of the knee joints of the supporting leg and swing leg, respectively; $\left(x_{2}, z_{2}\right)$ and $\left(x_{6}, z_{6}\right)$ denote the positions of the ankle joints of the supporting leg and swing leg, respectively; $\left(x_{7}, z_{7}\right)$, $\left(x_{4}, z_{4}\right)$ and $\left(x_{t}, z_{t}\right)$ denote the positions of the hip joint and the body, respectively; $l_{i}, i=1, \ldots, 7$ is the length of link $i$. 


\section{Formulation}

\section{1 Constraints}

Since the foot of swing leg must be on the ground both in the beginning and in the end of a stride period,

$$
\begin{aligned}
& z_{7}(0)=0 \\
& z_{7}\left(t_{f}\right)=0,
\end{aligned}
$$

where $t_{f}$ is the time at the end of the stride. At the end of a stride, the foot of the swing leg will be at distance $S$, which denotes the stride, away from its position at the beginning of the stride, which is mathematically expresses as

$$
x_{7}\left(t_{f}\right)-x_{7}(0)=S \text {. }
$$

Since locomotion is periodic, the speed and the configurations of the left and right legs at the end of the stride become identical to those of the right and left legs respectively at the beginning of the stride. Therefore,

$$
\begin{array}{ll}
\theta_{j}(0)=\theta_{7-j}\left(t_{f}\right), & (j=1,2, \ldots, 6) \\
\dot{\theta}_{j}(0)=\dot{\theta}_{7-j}\left(t_{f}\right), \quad & (j=1,2, \ldots, 6)
\end{array}
$$

Note that the foot of the swing leg shouldn't hit the ground until the end of a stride. In order to force this, the following inequality constraint condition should be met.

$$
z_{7} \geq \delta_{h} \quad\left(\text { when } t \neq 0, t_{f}\right),
$$

where $\delta_{h}$ is the minimum distance allowed to keep the foot off the ground during its swing motion.

In order to have a locomotion pattern similar to human's, where the knee joints are almost fully stretched at some point, and to avoid any singular kinetic configurations, two additional constrains are applied, one for each leg.

$$
\theta_{2} \leq \frac{\pi}{2}-\epsilon, \text { and } \theta_{5} \leq \frac{\pi}{2}-\epsilon \quad(\epsilon>0),
$$

where $\epsilon$ is the difference between the maximum allowable angle of the knees and the angle of the fully stretched leg, i.e., $\pi$.

When the zero-moment point (ZMP) moves around within a bound with in the foot print of the support leg, the robot seems to be stable, and

$$
\left\|x_{\mathrm{zmp}}\right\|<\frac{\Delta}{2}
$$

where $\Delta$ is the bound within which the ZMP should move, and

$$
x_{\mathrm{zmp}}=\frac{\sum_{i=1}^{6} m_{i}\left(\ddot{z}_{i}+g\right) x_{i}-\sum_{i=1}^{6} m_{i} \ddot{x}_{i} z_{i}}{\sum_{i=1}^{6} m_{i}\left(\ddot{z}_{i}+g\right)},
$$

and $\left(x_{i}, z_{i}\right)$ is the position of the mass center of link $i$.

\subsection{Design variables for optimization}

3.2.1 Basis functions to approximate the walking gait The trajectory of each joint angle is expressed as a 4 th-order polynomial of time $t$. Thus,

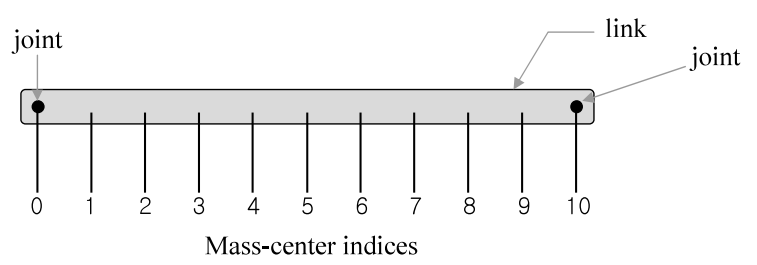

Fig. 2 Index for the mass-center of a link

$$
\begin{aligned}
& \theta(t)=A T \\
& \theta(t):=\left[\begin{array}{llllll}
\theta_{1} & \theta_{2} & \theta_{3} & \theta_{4} & \theta_{5} & \theta_{6}
\end{array}\right]^{T} \\
& A:=\left[a_{i, j}\right]_{i=1, \ldots, 6} j=1, \ldots, 5 \\
& T:=\left[\begin{array}{lllll}
1 & t & t^{2} & t^{3} & t^{4}
\end{array}\right]
\end{aligned}
$$

The coefficients of the polynomials representing all the joint angles, i.e., the components of matrix $A$, are used as design variables.

By the use of the kinetic constraints of Eqs. (13), (14) and (15), which represents 15 equations, the total number of the design variables is reduced from 30 to 15 .

\subsubsection{Optimal locations of the mass centers of} links More design variables added to the 15 design variables in order to search the optimal locations of the mass centers of links. Under the assumption that two legs have the identical static and dynamic characteristics, three variables are used to represent the mass center of the trunk link, thigh links, and shank links.

It is assumed that the mass center of a link cannot be located only at one of the 9 equally-spaced discrete points along the link, not including the extreme ends of the link. Each discrete point is represented by an index that changes from 1 to 9 as shown in Fig. 2. The indices for the both ends are 0 and 10. Thus, the actual optimal location of the mass center of link $i, x_{i}^{c g}$, is

$$
x_{i}^{c g}=\frac{k_{i}}{10} \cdot \ell_{i},
$$

where $k_{i}$ and $\ell_{i}$ respectively denote the mass center index and is the length of the link $i$.

Therefore, the total number of design variables is 18 , i.e., 15 for equality constraints and 3 for the mass center indices. This number is the length of the chromosome in the genetic algorithm to be used. As indicated already, a real number vector represents a chromosome. The indices for the mass centers is obtained rounding off the real numbers representing them.

\subsection{Cost function}

The performance index to be minimized is chosen as

$$
J(\alpha)=\frac{1}{2} \int_{0}^{t_{f}} p(\alpha)^{T} Q p(\alpha) d t
$$

where

$$
p(\alpha)=\left[\begin{array}{llll}
p_{1} & p_{2} & \ldots & p_{6}
\end{array}\right]^{T} \in \mathbb{R}^{6},
$$

and $p_{i}=u_{i} \dot{\theta}_{i}, i=1, \ldots, 6$ and $\alpha \in \mathbb{R}^{18}$ denote the motor power consumption at joint $i$ and the design variables, respectively; 


$$
Q=\operatorname{diag}\left(q_{1}, q_{2}, \ldots, q_{6}\right)>0 .
$$

whose elements are the weighting factors that represent the contributions by the joint motors to the cost function. Torque $u$ is derived from the inverse dynamics of the biped model:

$$
M(\theta) \ddot{\theta}+V(\theta, \dot{\theta})+G(\theta)=u
$$

The inequality constraints in Eqs. (18), (19), and (20) can be expressed as

$$
g(\alpha)=\left[\begin{array}{c}
\delta_{h}-z_{7} \\
\theta_{2}-\frac{\pi}{2}+\epsilon \\
-\theta_{5}-\frac{\pi}{2}+\epsilon \\
\left\|x_{\mathrm{zmp}}\right\|-\frac{\Delta}{2}
\end{array}\right] \leq 0 \in \mathbb{R}^{4} .
$$

A transformation method convert a constraint optimization problem into an unconstrained problem with a transformation function of

$$
F(\alpha, r)=J(\alpha)+P(g(\alpha), r)
$$

where $r$ is a vector of penalty parameters and $P$ is a real valued function whose action of imposing the penalty is controlled by $r$. The form of penalty function $P$ depends on the transformation method used. Here, the following exterior penalty function method is used.

$$
P(g(\alpha), r)=\sum_{j=1}^{3} r_{j}\left[g_{j}^{+}(\alpha)\right]^{2}
$$

where $g_{j}^{+}(\alpha)=\max \left(0, g_{j}(x)\right)$, and $r_{j}$ is a weight. The value of function $g_{j}^{+}(\alpha)$ is zero if its corresponding constraint is satisfied, i.e., $g_{j}(\alpha)<0$, and is positive otherwise, i.e. $g_{j}(\alpha) \geq 0$.

\subsection{Optimization with genetic algorithm}

Genetic algorithms are used in many optimization problems for the reasons that they are robust to illcondition in the optimization function and that they can find global solutions. A genetic algorithm operates through a simple flow of four processes. At the first process, an initial population is created as a starting point for the search. In the next process, each individual is evaluated for its fitness. Based on the relative fitness of the individuals, some individuals in the population are selected for reproduction. In the reproduction, a crossover operator takes two chromosomes and swaps parts of their genetic information to produce new chromosomes. A mutation operator also produces new genetic structures in the population by randomly modifying some of the genes, helping the search algorithm escape from a local minimum. The offsprings produced by these genetic processes become the next population to be evaluated. These processes are repeated until a satisfactory individual is found or other a certain stop condition is met.

In this work, a real-coded genetic algorithm is used because binary-coded genetic algorithms have many problems in the practical applications to my problems ${ }^{(14)}$. A real encoding has a few advantages over a binary encoding. First, programming is simple and searching speed is improved since the encoding and decoding the processes is not needed due to the one-to-one correspondence between a phenotype and a genotype. Second, it is possible to define a very large domain and easy to deal with highly complex constraints.

In this paper, among many different operators, the modified simple crossover and the boundary mutation are used since the former is a operator made up for the weak points of the simple crossover by using the method of the arithmetical crossover, and the latter can make large variations in genes.

For each member of the trajectory population, the dynamics of the biped robot of Eq. (28) is simulated for a single step. The value of the cost function computed based on the data from the simulation is used to evaluate the goodness of the trajectory. Few best members of the population then participate in producing their off-springs, i.e., robot trajectories, through cross-over and mutations.

\section{Simulations}

\subsection{The trajectory based on the gravity- compensated inverted pendulum model}

A simulation of biped-robot locomotion is done based on the leg trajectory generated by the GCIPM, which is based on the foot trajectory of the swing leg:

$$
\begin{aligned}
& x(t)=-S \cos \left(\pi \frac{t}{t_{f}}\right) \quad\left(0 \leq t \leq t_{f}\right) \\
& z(t)=\frac{h_{f}}{2}\left[1-\cos \left(2 \pi \frac{t}{t_{f}}\right)\right] \quad\left(0 \leq t \leq t_{f}\right)
\end{aligned}
$$

where $h_{f}, t_{f}$ denote the maximum height of the swing leg and the time span for a single stride, respectively. In the simulation, $h_{f}=0.10 \mathrm{~m}, S=0.3 \mathrm{~m}, t_{f}=1.0 \mathrm{~s}$, and $\Delta=0.18 \mathrm{~m}$. Note that the foot trajectory is represented concisely by sinusoidal functions of time $t$. Figure 3 shows the stick diagram of the biped robot.

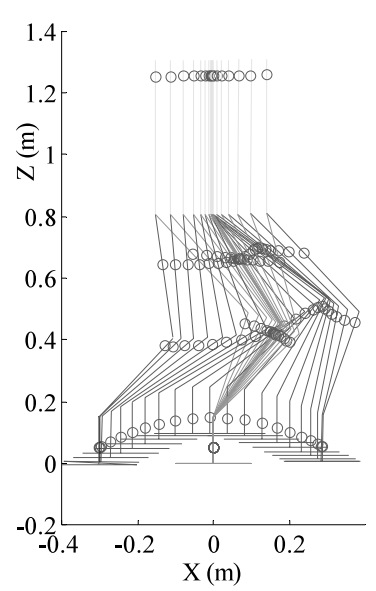

Fig. 3 Stick diagram of the robot locomotion based on the GCIPM 
Table 2 Parameters used in the genetic algorithm with the predetermined mass centers

\begin{tabular}{cc}
\hline Parameters & values \\
\hline Max. generations & 3,000 \\
Population & 30 \\
Chromosome length & 15 \\
Crossover ratio & 0.9 \\
Mutation ratio & 0.02 \\
\hline
\end{tabular}
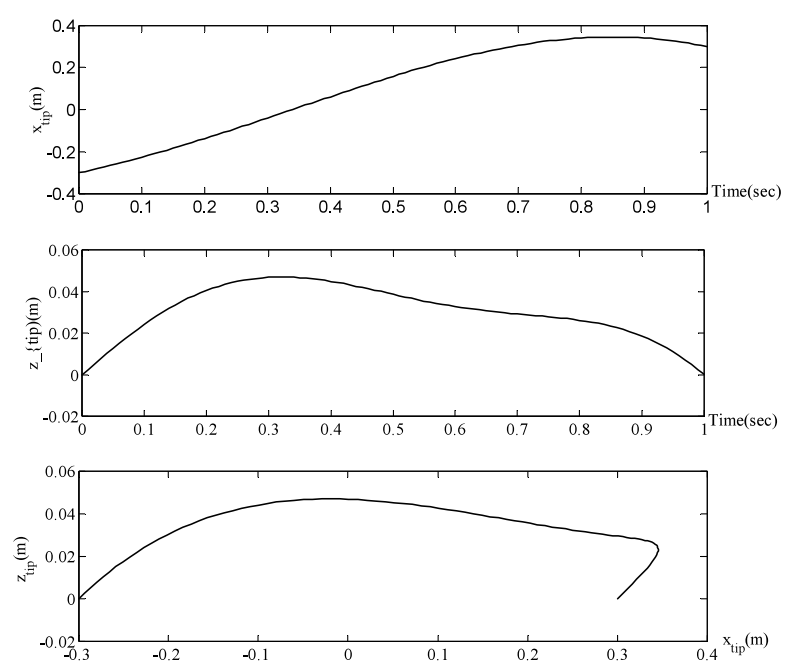

Fig. 4 The trajectory of the foot of the swing leg generated by the GA with predetermined mass centers

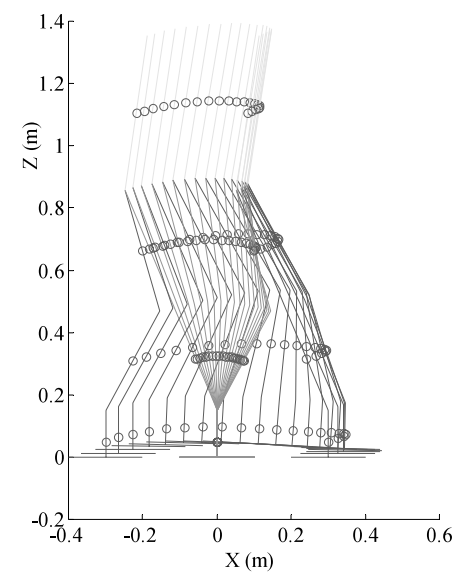

Fig. 5 Stick diagram of the robot locomotion generated by the GA with predetermined mass centers

\subsection{Optimal locomotion trajectory with predeter- mined mass-centers}

In this simulation, it is assumed that the center of gravity of each link is known and fixed at the center of the link. The parameters used in the genetic algorithm are listed in Table 2. If the number of generation reaches the maximum number of generation set by the software or if the value of the cost function does not change for 50 consecutive generations, the simulation is terminated. Under

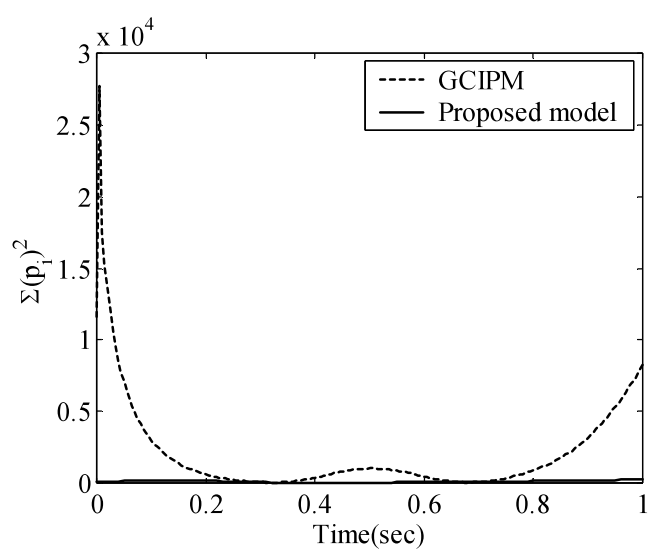

Fig. 6 Comparison of the power consumption in the locomotion generated by the GCIPM and the GA with the predetermined mass centers

Table 3 Parameters used in the genetic algorithm with the mass centers to be optimized

\begin{tabular}{cc}
\hline Parameters & values \\
\hline Max. generations & 3,000 \\
Population & 30 \\
Chromosome length & 18 \\
Crossover ratio & 0.9 \\
Mutation ratio & 0.02 \\
\hline
\end{tabular}

these conditions, it takes about 1870 generations to obtain a reasonable result. Figures 4 and 5 show a stick-diagram and the trajectory of the foot of the swing leg. They indicate that the optimal locomotion pattern is very similar to that of a human. Figure 6 shows that the power consumption level and thus the energy consumption of the trajectory generated by this method are far less than those of the trajectory generated by the GCIPM.

\subsection{Optimal locomotion trajectory}

In this simulation, it is assumed that the center of gravity of each link is not fixed and is treated as an extra variable to be optimized. Table 3 shows the simulation conditions used in the GA. Under the same terminating conditions as in section 4.2, it takes about 1600 generations to obtain a reasonable result. Figure 7 shows the performance of the cost function as the generation progresses. Figure 8 shows the optimal trajectory at the joint level. Figure 9 shows the stick diagram of the biped robot based on it. Figure 10 shows that the trajectory of the foot of the swing leg is similar to that of a human as in the case with the predetermined mass centers. Figure 11 indicates how the indices of the optimal mass centers, which indicates the optimal locations of the mass centers of the links, are searched for by the proposed method. The optimal location of the mass center of each shank is near the knee (optimal index $=0.9$ ). For each thighs, the optimal location of the mass center is near but slightly higher 


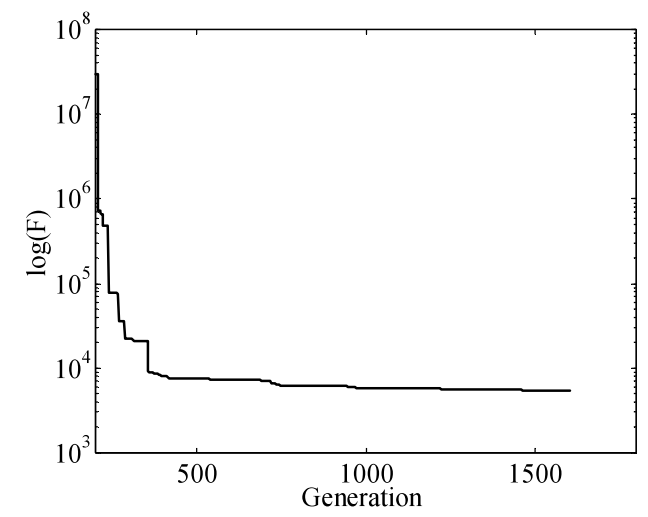

Fig. 7 The minimum value of the cost function versus the generation

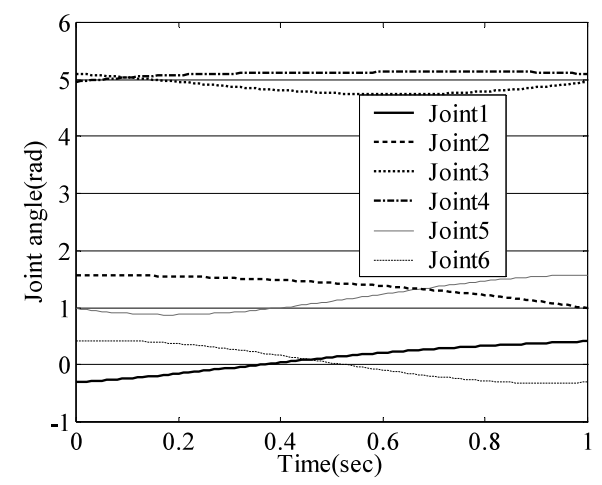

Fig. 8 Joint trajectory of the robot generated by the GA with the optimal mass centers

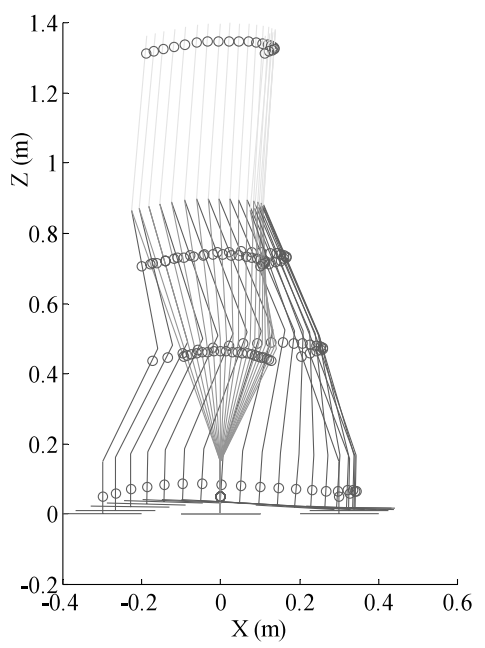

Fig. 9 Stick diagram of the robot locomotion generated by the GA with the optimal mass centers

than the center of its length (optimal index $=0.6$ ). For the trunk, its optimal location of the mass center is near the distal end (optimal index $=0.9$ ). The optimal locations of the mass centers are graphically represented as the small circles in Fig. 9. Figure 12 shows how the ZMP changes in the optimal locomotion. Figure 13 shows that the total power level is lower when the mass centers are not predetermined. By selecting the optimal positions of the
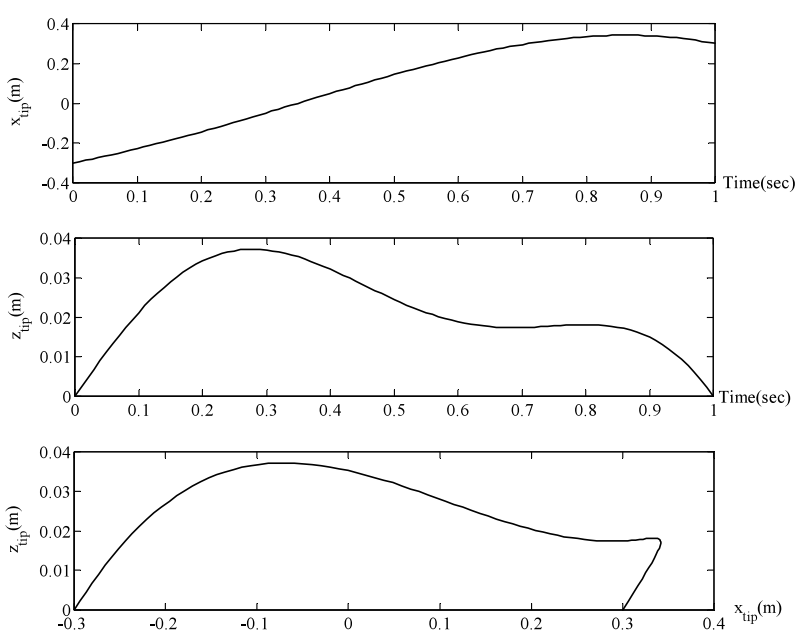

Fig. 10 The trajectory of the foot of the swing leg generated by the GA with the optimal mass centers

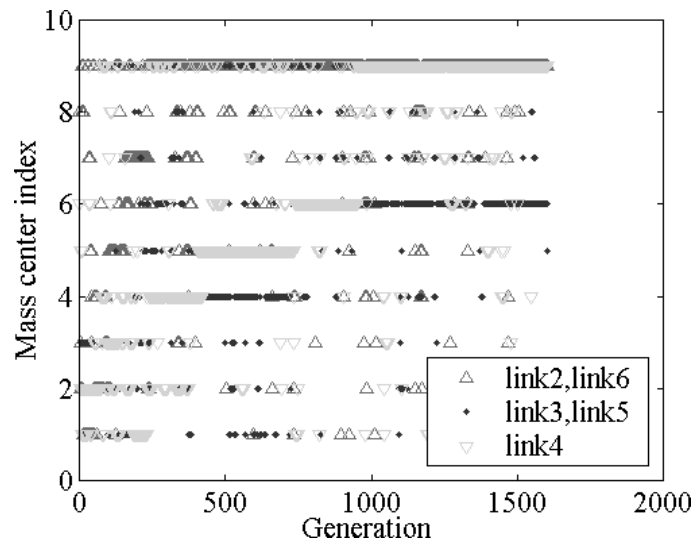

Fig. 11 Changes in the mass center indices as the generation progresses

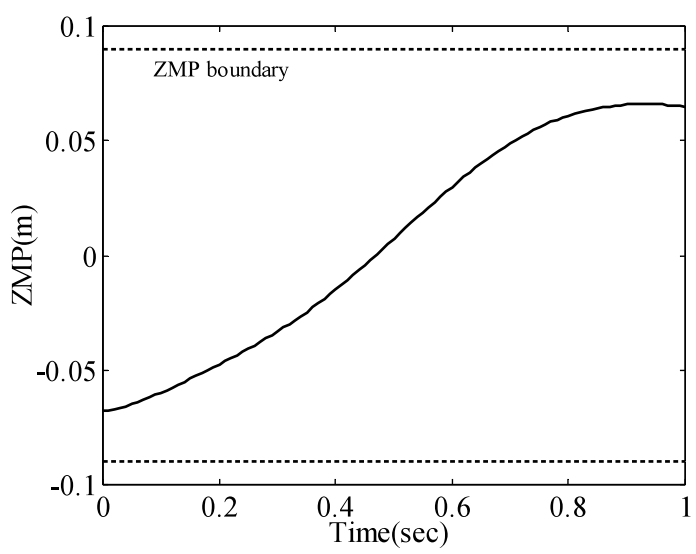

Fig. 12 ZMP position

mass centers, the energy consumption is further reduced by $83.8 \%$ and $26.8 \%$ compared with the GCIPM and the GA with predetermined mass-centers, respectively.

\section{Conclusions}

This paper proposes a method that minimizes the energy consumption by finding the optimal locations of the 


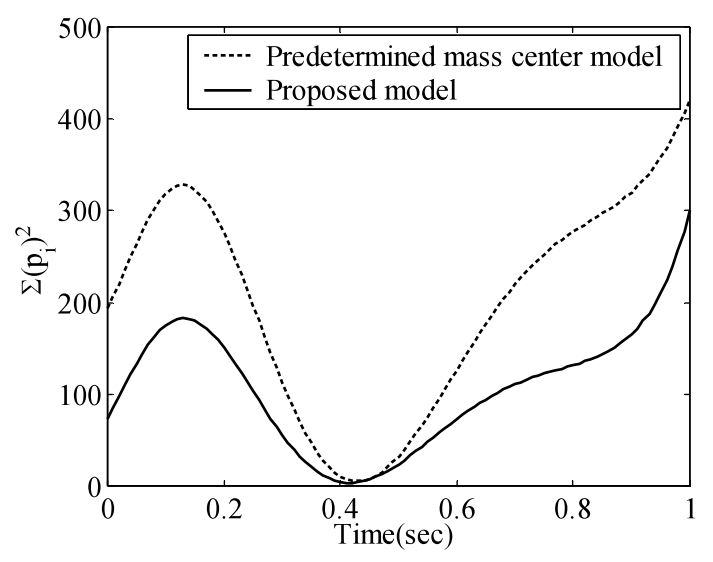

Fig. 13 Comparison of the power consumption in the locomotion generated by the GA with the predetermined mass centers and with the optimal mass centers

mass centers of the links, and the optimal trajectory of the legs using a real-coded genetic algorithm. In order to find out the optimal trajectory, the coefficients of 4th order polynomials and the indices of the mass centers are used as design variables. Simulation results indicate that the GA solution with the optimal mass centers offers $83.8 \%$ and $26.8 \%$-reduction in the energy consumption compared respectively with the GCIPM and the GA with the predetermined mass centers of the links.

\section{References}

( 1 ) Hasegawa, Y., Arakawa, T. and Fukuda, T., Trajectory Generation for Biped Locomotion Robot, Mechatronics, Vol.10, No.1-2 (2000), pp.67-89.

( 2 ) Hreljac, A. and Martin, P.E., The Relationship between Smoothness and Economy during Walking, Biological Cybernetics, Vol.69, No.3 (1993), pp.213-218.

( 3 ) Kajita, S. and Tani, K., Experimental Study of Biped Dynamic Walking in the Linear Inverted Pendulum Mode, IEEE Int. Conf. on Robotics and Automation, Nagoya, Japan, (1995), pp.2885-2891.
( 4 ) Park, J.H. and Kim, K.D., Biped Robot Walking Using Gravity-Compensated Inverted Pendulum Mode and Computed Torque Control, IEEE Int. Conf. on Robotics and Automation, Lueven, Belgium, (1998).

( 5 ) Lee, T.T. and Chen, Y.C., Minimum-Fuel Path Planning of a 5-Link Biped Robot, Southeastern Symp. on System Theory, (1988), pp.459-463.

( 6 ) Roussel, L., de Wit, C.C. and Goswami, A., Comparative Study of Method for Energy-Optimal Gait Generation for Biped Robots, Int. Conf. on Informatics and Control, (1997), pp.1205-1212.

( 7 ) Roussel, L., de Wit, C.C. and Goswami, A., Generation of Energy Optimal Complete Gait Cycles for Biped Robots, IEEE Int. Conf. on Robotics and Automation, Leuven, Belgium, (1998), pp.2036-2041.

( 8 ) Westervelt, E.R. and Grizzle, J.W., Design of Asymptotically Stable Walking for a 5-Link Planar Biped Walker via Optimization, IEEE Int. Conf. on Robotics and Automation, Washington D.C., USA, (2002), pp.3117-3122.

(9) Capi, G., Kaneko, S., Mitobe, K., Barolli, L. and Nasu, Y., Optimal Trajectory Generation for a Prismatic Joint Biped Robot Using Genetic Algorithms, Robotics and Autonomous Systems, Vol.38, No.2 (2002), pp.119128.

(10) Silva, F.M. and Machado, J.A.T., Dynamic Performance of Biped Locomotion Systems, Int. Workshop on Advanced Motion Control, (1998), pp.451-456.

(11) Chevallereau, C., Formal'sky, A. and Perrin, B., Low Energy Cost Reference Trajectories for a Biped Robot, IEEE Int. Conf. on Robotics and Automation, Leuven, Belgium, (1998), pp.1398-1404.

(12) Gen, M. and Cheng, R., Genetic Algorithms and Engineering Optimization, (2000), Wiley.

(13) Srinivas, M. and Patnaik, L.M., Genetic Algorithms: A Survey, Computer, Vol.27, No.6 (1994), pp.17-26.

(14) Jin, G.G., Genetic Algorithms and Their Applications, (2002), Kyowoo-Sa.

(15) Furusho, J. and Sano, A., Sensor-Based Control of a Nine-Link Biped, Int. J. of Robotics Research, Vol.9, No.2 (1990), pp.83-98. 